\title{
Electrodermal Activity: Explorations in the Psychophysiology of Engagement with Social Robots in Dementia*
}

\author{
Giulia Perugia, Daniel Rodríguez-Martín, Marta Díaz Boladeras, Andreu Català Mallofré, Emilia \\ Barakova, and Matthias Rauterberg
}

\begin{abstract}
The study of engagement is central to improve the quality of care and provide people with dementia with meaningful activities. Current assessment techniques of engagement for people with dementia rely exclusively on behavior observation. However, novel unobtrusive sensing technologies, capable of tracking psychological states during activities, can provide us with a deeper layer of knowledge about engagement. We compared the engagement of persons with dementia involved in two playful activities, a game-based cognitive stimulation and a robot-based free play, using observational rating scales and electrodermal activity (EDA). Results highlight significant differences in observational rating scales and EDA between the two activities and several significant correlations between the items of observational rating scales of engagement and affect, and EDA features.
\end{abstract}

\section{INTRODUCTION}

Dementia is a neurodegenerative disorder whose prevalence rates are continuously growing and will reach 115 million people by 2050 [1]. As a disease, dementia affects cognition, producing a reduction in thinking, problem solving and mnemonic ability, functioning, preventing affected people to care for themselves and carry out activities of daily living (e.g. self-feeding), and psychological wellbeing, causing the appearance of disorders of perception, thought content, mood, and behavior called behavioral and psychological symptoms of dementia (BPSD; e.g. apathy, depression, agitation, anxiety) [2].

People with dementia living in institutionalized contexts spend most of their time inactive [3]. However, several studies have demonstrated the importance of participation in activities to improve quality of life (QoL) and wellbeing in dementia $[4][5][6][7][8]$. In this respect, social robots are

\footnotetext{
* Erasmus Mundus Joint Doctorate (EMJD) in Interactive and Cognitive Environments (ICE), funded by Erasmus Mundus under the FPA no. 20102012.

G. Perugia is with the Eindhoven University of Technology, Eindhoven, Netherlands, 5612 AZ (e-mail: g.perugia@tue.nl).

D. Rodríguez-Martín is with the Technical University of Catalonia, Vilanova i la Geltrú, Barcelona, Spain, 08800 (e-mail: daniel.rodriguezmartin@upc.edu)

M. Díaz Boladeras is with the Technical University of Catalonia, Vilanova i la Geltrú, Barcelona, Spain, 08800 (e-mail: marta.diaz@upc.edu).

A. Català Mallofré is with the Technical University of Catalonia, Vilanova i la Geltrú, Barcelona, Spain, 08800 (e-mail: andreu.catala@upc.edu).

E. Barakova is with the Eindhoven University of Technology, Eindhoven, Netherlands, 5612 AZ (e-mail: e.i.barakova@tue.nl).

M. Rauterberg is with the Eindhoven University of Technology, Eindhoven, Netherlands, 5612 AZ (e-mail: g.w.m.rauterberg@tue.nl).
}

deemed useful to improve positive affect [9] and social interactions and to reduce loneliness [10][11].

A crucial node when discussing about participation in activities is their meaningfulness for the person with dementia. In this sense, it is decisive to measure, together with the long-term impact of activities on health, cognition, and psychosocial wellbeing, also the inherent engagement state that activities are able to foster in people with dementia. We define engagement as the psychological state of wellbeing, enjoyment and active involvement that is triggered by meaningful activities and causes people to be enraptured by the activity (thus more resistant to distraction), more energetic (thus more prone to work to achieve their objectives and less inclined to feel the effort), and in a more positive mood.

To pursue a comprehensive measurement of engagement for dementia, state of the art assessment techniques are insufficient. Indeed, most of them rely exclusively on behavior observation [12][13], but behavior can be misleading in dementia since the display of inner states can be impaired (i.e. apathy). New miniaturized sensing technologies, able to gauge the psychophysiology of engagement can come in handy to enrich the knowledge drawn from behavior observation. For instance, electrodermal activity (EDA) can be measured in an unobtrusive way with wearable sensors and gives a straightforward measure of arousal. EDA is a change in the skin conductance that derives from the activation of the sympathetic nervous system in response to episodes of excitement, attention, anxiety, and high cognitive load [14].

In this paper we present a study aimed at comparing the engagement of persons with dementia involved in two activities: a game-based cognitive stimulation and a robotbased free play. The two proposed activities were assumed to elicit different types of engagement, cognitive and affective. We examined the underlying arousal states that the activities triggered, and studied their relationships with observed behavior. As a result, we present clear directions to follow in the understanding of the psychophysiology of engagement in dementia.

\section{RELATED WORK}

Ideally, related work would include studies that gauged EDA to understand and profile the quality of interactions with social robots of persons with dementia. However, in spite of the great interest of the Human-Robot Interaction (HRI) community for the therapeutic effects of social robots on dementia patients, studies on the physiology of engagement with social robots of people with dementia are scarce and involve costly and invasive procedures, such as 
EEG [15], urinalysis and hormones analysis [16], and fNIRS [17].

In the context of dementia, we found only two studies conducted with EDA, both for purposes beyond our interest [18][19]. The only real antecedent of the present study is a study carried out with elders with Mild Cognitive Impairment (MCI) during interactions with a telepresence robot (Giraff) using cardiac measures, heart rate (HR) and heart rate variability (HRV) [20].

As a consequence, the closest reference to our work is research with healthy adults and children. In this area, studies on EDA could be divided into two typologies: hypothesistesting and affect recognition studies. On one hand, hypothesis-testing studies are works focusing on statistical analyses aimed at identifying differences in EDA between experimental conditions and at detecting correlations between self-report questionnaires and EDA features. On the other hand, affect recognition studies are works where EDA features are used to classify diverse affective states with a certain degree of accuracy. In HRI, most studies focused on affect recognition. For instance, Henriques et al. (2013) [21] worked on EDA-based recognition of empathy, expectation, positive-surprise, stress, and frustration in two conditions, human-to-human and human-to-robot interaction (with NAO), and Leite et al. (2013) [22] investigated the benefits of measuring EDA to adapt online the behavior of iCat according to children's emotional and cognitive processes. However, hypothesis-testing studies are also available. Rosenthal-von der Pütten et al. (2012) [23] used EDA and self-reported emotions to evaluate affective reactions towards videos depicting the dinosaur robot Pleo in two situations, a friendly and a torturing interaction. In our case, given the lack of literature coverage on EDA in people with dementia, we could not yet aim at affect recognition, thus we used EDA to differentiate between different types of engagement and worked on the identification of trends in the EDA data by correlating EDA features with the items of observational rating scales of engagement.

An additional problem to face when measuring EDA in vulnerable participants is caused by the reactivity to the experimental situation. Indeed, the artificiality of the situation might prompt physiological responses not linked with the experimental conditions (e.g. anxiety), thus jeopardizing the reliability of the data collected. In recent years, the development of wearable and unobtrusive devices aimed at collecting physiological data in real life settings (e.g. Q sensor and E4 wristband ${ }^{1}$ ) paved the way for in the field data collection. In this regard, Leite and colleagues [22] were among the first to seize the opportunity, collecting children's EDA and HR at school with the Q sensor. In the attempt to follow this ecologically valid approach, we collected EDA directly in nursing homes using the more advanced E4 wristband.

The following sections detail the research design of the study and the procedures used to collect EDA (Section III), the results obtained from analyses of observational rating scales and EDA (Section IV), and the main conclusions drawn from the results (Section V).

\footnotetext{
${ }^{1}$ https://www.empatica.com/e4-wristband
}

\section{METHOD}

\section{A. Aim}

The present study was carried out to: 1) study the different engagement and arousal states that the two proposed activities brought about in people with dementia using observational rating scales and EDA, and 2) uncover correlations between participants' physiological states and observed engagement in activities.

\section{B. Participants}

Fourteen participants aged between 69 and 92 years $(M$ : $83.93, S D: 7.28$ ) with a diagnosis of dementia ranging from mild to moderate were selected from two nursing homes in the province of Barcelona. Selection was performed together with the psychologists of the two nursing homes. Dementia severity was assessed using the Reisberg Global Deterioration Scale (GDS) [24]. Subjects included in the study had a confirmed dementia diagnosis and a deterioration level ranging from mild to moderate (scores 4 and 5 of the Reisberg GDS). Exclusion criteria were a diagnosed bipolar or schizophrenic disorder, abnormality in the movement of face or hands (e.g. Parkinson's disease), strong hallucinatory states, and bedridden condition.

Selected participants were randomly coupled, and took part in the activities in pairs. This was in order to reproduce as closely as possible the usual social context of activities in nursing homes, which is group-based.

\section{Design}

The study was conducted in nursing homes and followed a repeated measurement design with two playful activities as experimental conditions: a game-based cognitive stimulation, and a robot-based free play. Participants took part in the two activities once per week. Each activity was presented three times, for a total of six sessions per couple, three of cognitive games and three of robot play. The order of presentation of activities was alternated: cognitive games and robot play were presented every other week starting from cognitive games.

Cognitive games were jigsaw puzzles, shape puzzles, and dominoes (Figure 1). The order of presentation of the cognitive games was randomized using a Latin squares technique, thus it was always different in the three sessions.

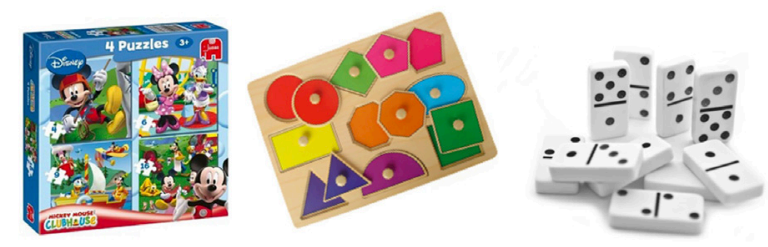

Figure 1. Cognitive Games

For what regards the robot play, we left participants free to interact with the robot, Pleo (Figure 2), and gave facilitators a script with a list of activities to be introduced in case the interaction faded (call Pleo, make Pleo sleep, feed Pleo, dress Pleo, stroke Pleo, heal Pleo).

Pleo is an animatronic pet robot commercialized by UGOBE, which has the appearance of a baby dinosaur. It is 
equipped with touch sensors, microphones, ground foot sensors, force-feedback sensors, orientation tilt sensors, infrared mouth sensors, a camera-based vision system, and a beat detection system.

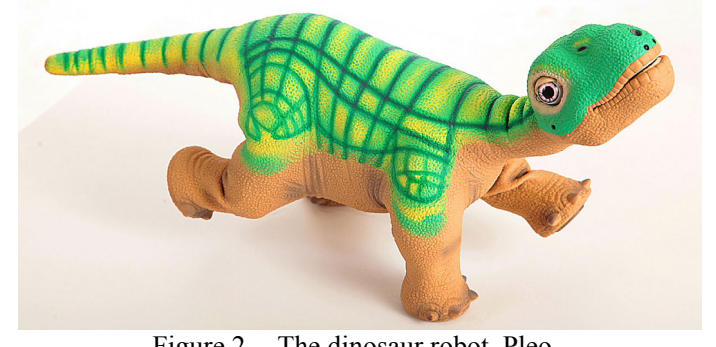

Figure 2. The dinosaur robot, Pleo

Pleo is not only able to display a wide range of behaviors (e.g. walk, howl), but also to express its internal drives (e.g. hunger or sleep) and moods (e.g. happy, scared, curious). We selected this robot, instead of other available ones, because, being designed for children, it provides a very prompt interaction, and this is very important when working with people with mild to moderate dementia who are still able to sustain dynamic exchanges.

The two experimental conditions we chose are not canonical. Usually, interactions with social robots are compared to interactions with stuffed animals or dolls. We chose the present experimental conditions for three reasons: a) a stuffed animal/doll condition is risky with people with mild dementia who are still able to make sense of the world and might feel infantilized; b) reviews on the use of social HRI for people with dementia deplore the fact that most studies use experimental and control conditions that are likely to produce the same effects (e.g. dolls and social robots) [25]; c) the chosen experimental conditions hinge upon different skills: cognition the cognitive games, and affect the interactions with the social robot, which both elicit arousal, but of a different type.

During sessions, four facilitators followed the participants (the psychologist or the social educator of each nursing home). Facilitators were coupled randomly with participants, and followed the same couple/s across all sessions.

\section{Measures}

In total, we carried out forty-two play sessions of around half an hour each. During sessions, we collected three types of data: video footage, physiological data, and observational rating scales.

Video footage of all the sessions was collected with two hand held cameras positioned on mini-tripods. Videos were used to label the physiological signals.

Physiological data were collected using the E4 wristband [26]. The E4 wristband has four sensors integrated in its case: a photoplethysmography sensor (to measure heart rate, heart rate variability, and inter-beat interval), a triaxial accelerometer (to measure acceleration, but also to detect movement patterns), an infrared thermometer (to gauge peripheral skin temperature), and an electrodermal response sensor (to assess arousal). We recorded data from all the sensors of the E4 wristband. However, in this paper, we analyze those coming from the electrodermal response sensor.

The EDA signal was synchronized with video footage to establish the beginning and end of baseline collection, and the beginning and end of activities. Subsequently, it was normalized and denoised with a $2^{\text {nd }}$ order Butterworth lowpass filter with a cut-off frequency of $0.05 \mathrm{~Hz}$. Regarding the extracted features, we used differential features in order to take into account the baseline state of the person with dementia in the calculation of EDA.

The feature notation in Table 1 is constructed in the following way. The set of samples $S^{W}=s_{1}^{W}, \ldots s_{N}^{W}$ is recorded in a window of time $W$ defined by the beginning and the end of an activity (cognitive games or robot play). The Short Fast Fourier Transform of this sample set was formed by $S_{1}^{W}, \ldots S_{N}^{W}$ through (1):

$$
S_{h}^{W}=\sum_{n=1}^{N} S_{n} e^{-i 2 \pi h \frac{n}{N}}
$$

where $h=1, \ldots, N . S_{h}^{W}$ is a set of $N$ complex numbers that represents the amplitude and phase of a harmonic. In regards to Npeaks, we denoted it as the number of significant local maxima found in $S^{W}$. Then, $N P R^{W}$ is defined as $\operatorname{Npeaks}\left(S^{W}\right)$ divided by the length of $S^{W}$.

TABLE 1. Feature set

\begin{tabular}{|c|c|}
\hline Feature name & Equation \\
\hline $\operatorname{diffSMA}^{\mathrm{a}}$ & $\int_{i=0}^{T} s_{i}^{W} d t-\int_{i=0}^{T} s_{i}^{W-1} d t$ \\
\hline $\operatorname{diffM}^{b}$ & $s^{W}-\bar{s}^{W-1}$, where $\bar{s}^{W}=\frac{1}{N} \sum_{i=1}^{N} s_{i}^{W}$ \\
\hline $\operatorname{diffSD}^{c}$ & $\sigma S^{W}-\sigma S^{W-1}$, where $\sigma S^{W}=\sqrt{\frac{1}{N} \sum_{i=1}^{N}\left(s_{i}^{W}-\bar{s}^{W}\right)^{2}}$ \\
\hline $\operatorname{diffRNG}^{\mathrm{d}}$ & $\begin{array}{c}\operatorname{Rng}\left(s^{W}\right)-\operatorname{Rng}\left(s^{W-1}\right), \text { where Rng }\left(s^{W}\right) \\
=\max \left(s^{W}\right)-\min \left(s^{W}\right)\end{array}$ \\
\hline $\operatorname{diffSH}{ }^{\mathrm{e}}$ & $\sum_{i=1}^{N} S_{1, \mathrm{~N}}^{W}$ \\
\hline $\operatorname{diffNPR}^{\mathrm{f}}$ & $N P R^{W}-N P R^{W-1}$ \\
\hline diffKURT $^{\mathrm{g}}$ & $\delta_{s}^{W}-\delta_{s}^{W-1}$, where $\delta_{z}^{W}=\frac{\mathrm{E}\left[\left(\left|S_{1, N}^{W}\right|_{i}-\left|\bar{S}_{1, N}^{W}\right|\right)^{4}\right]}{\left(\mathrm{E}\left[\left(\left|S_{1, N}^{W}\right|_{i}-\left|\bar{S}_{1, N}^{W}\right|\right)^{3}\right]\right)^{2}}$ \\
\hline diffSKEW ${ }^{\mathrm{h}}$ & $\gamma_{s}^{W}-\gamma_{s}^{W-1}$, where $\gamma_{s}^{W}=\mathrm{E}\left[\left(\frac{\left|S_{1, N}^{W}\right|_{i}-\left|\bar{S}_{1, N}^{W}\right|}{\sigma\left(\left|S_{1, \mathrm{~N}}^{W}\right|\right)}\right)^{3}\right]$ \\
\hline $\begin{array}{l}\text { a. } \text { diffSMA }=\text { differ } \\
\text { differential standard } \\
\text { summation of harm } \\
\text { differential kurtosis; }\end{array}$ & $\begin{array}{l}\text { nal magnitude area; b. diffM= differential mean; c. diffSD= } \\
\text { on; d. diffRNG }=\text { differential range; e. diffSH= differential } \\
\text { diffNPR }=\text { differential number of peaks ratio; g. diffKURT= } \\
\mathrm{EW=} \text { differential skewness }\end{array}$ \\
\hline
\end{tabular}

As observational rating scales, we used the Observational Measurement of Engagement (OME) [12], and enriched it with the Observed Emotion Rating Scale (OERS) [13]. The OME rates engagement across four dimensions: duration of interaction (in seconds), attention towards the stimulus (e.g. manipulating/holding the stimulus, 4-point Likert scale), attitude towards the stimulus (e.g. smiling/frowning; 7-point Likert scale), and refusal of the stimulus. In our study, we used the items attention (4-points Likert scale) and attitude (7-point Likert scale) of the OME, using the latter to obtain scores of the attitude of participants towards the game, and 
towards the partner. Moreover, we added a further item, cognitive difficulty (5-point Likert scale), which is present in further versions of the OME. The OERS was used in its original version to rate (on a 5-point Likert) the intensity of five affective states: pleasure (e.g. laughing, smiling, kissing), anger (e.g. yelling, cursing, berating), anxiety/fear (e.g. shrieking, repetitive calling out), sadness (e.g. crying, frowning), and alertness (e.g. participating in a task, maintaining eye contact). Both the OME and the OERS were developed for psychogeriatric purposes, and are validated observational scales. They were completed after sessions by facilitators. For what concerns cognitive games, we asked facilitators to fill out one OME and one OERS for each of the games that were played. As for robot play, we obtained one OME and one OERS for the entire session.

\section{E. Setting}

Data were collected directly in the nursing homes, in rooms normally allocated to activities. The rooms in the two nursing homes were adapted to resemble each other. A rectangular table was placed on one side of the room, and on the opposite side we positioned two hand-held cameras on top of portable tripods (Figure 3). One camera was positioned on the side, and the other in front of the participants.

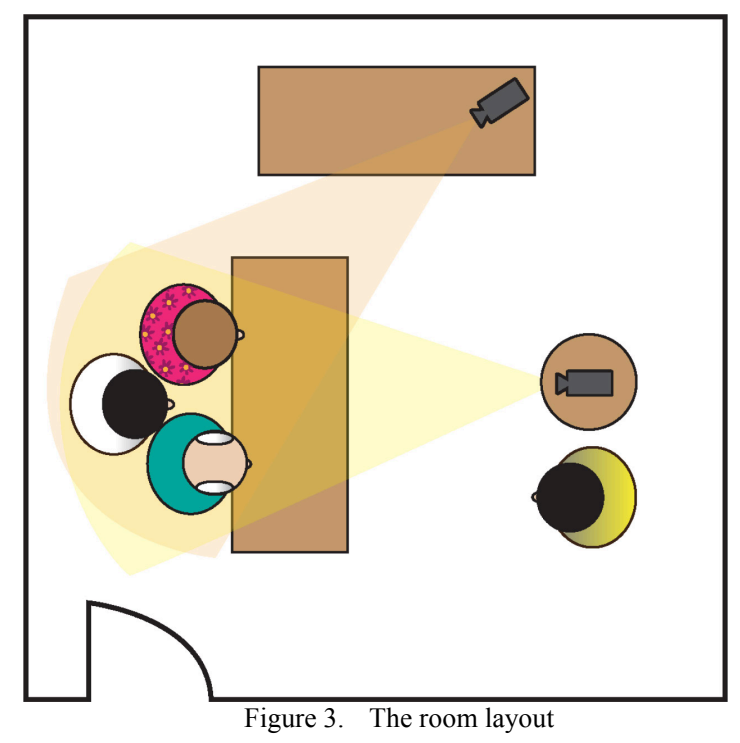

During the data collection participants were sitting on the same side of the table facing the cameras. The facilitator conducted the activity in the presence of the experimenter. The experimenter was present during data collection to supervise the correct functioning of the equipment (cameras and wristbands), to control that playful activities were correctly executed, and to collect the OME and OERS at the end of the sessions. However, to avoid its presence to be disruptive for participants, before the study started, the experimenter was introduced to participants and clients of the nursing homes, and took part in the activities promoted in the two institutions for few weeks.

\section{F. Procedure}

During the sessions, data collection was performed in six phases: 1) preparation phase: the experimenter set up the room, while the facilitator helped participants to reach it, once participants reached the room, cameras were switched on; 2) habituation phase: the experimenter conversed shortly with participants, while they sat to recover from the effort of walking to reach the room, afterwards the experimenter helped participants to wear the sensor (Figure 4a); 3) synchronization: the experimenter switched on the wristbands, and pushed the tag buttons on top of the watches simultaneously in front of the switched on cameras; 4) baseline collection: the facilitator read a fairytale ( 5 minutes) to participants (Figure 4b); 5) data collection: the participants played the three cognitive games (20-25 minutes, Figure 4c and 4d) or interacted with Pleo (20-25 minutes, Figure 4e and 4f); 6) end of activity: the experimenter switched off the wristbands in front of the cameras, removed them, and switched off the cameras. At this point, participants were guided back to their units, and, when facilitators headed back to the activity room, they filled out the OME and OERS.
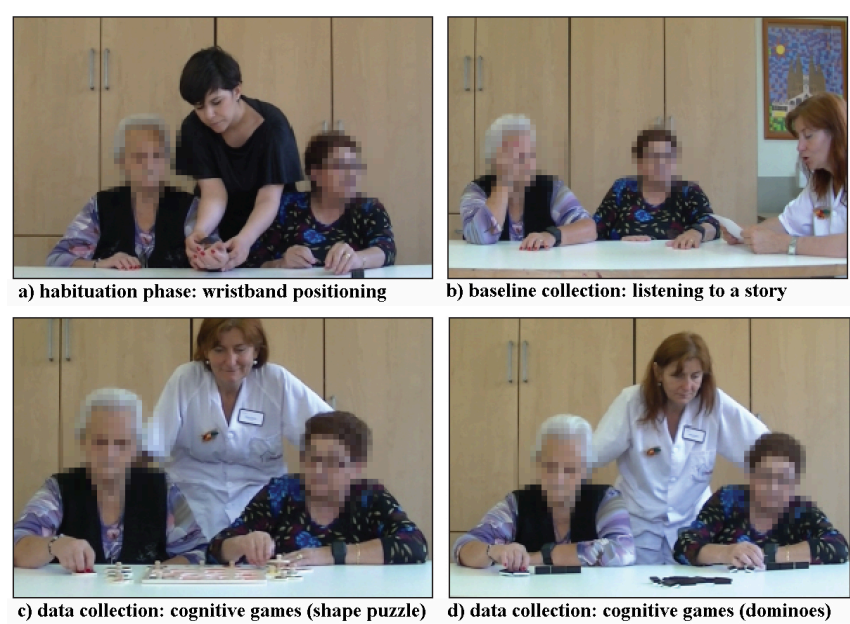

b) baseline collection: listening to a story
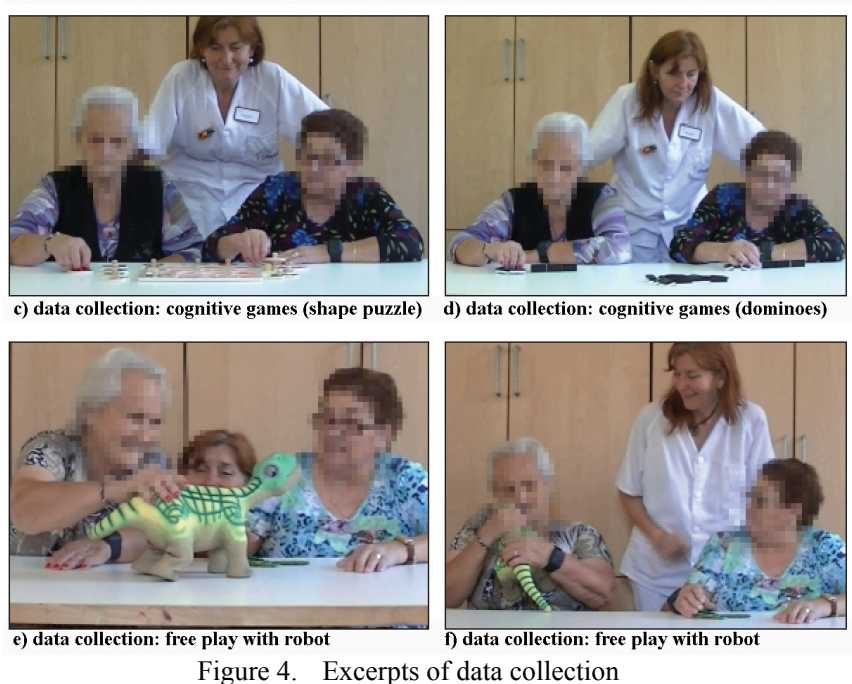

f) data collection: free play with robot

Figure 4. Excerpts of data collection

\section{G. Research Questions and Hypotheses}

The research questions we investigated with this study were the following: 1) Are there differences in engagement between the two activities as measured with OME and OERS? 2) Are there differences in arousal between the two activities as measured with EDA? and 3) Are there significant correlations between the items of OME and OERS and EDA features?

With regard to the first question, we expected that the different engagement states that the two activities elicited would have been mirrored by differences in the item cognitive difficulty of the OME and pleasure of the OERS. In detail, we hypothesized that cognitive difficulty would have been higher during cognitive games, while pleasure would have been higher during robot play. 
For what concerns EDA, we hypothesized that there would have been differences in EDA features due to the fact that the two activities both elicited arousal, but of a different type. Indeed, Cannon [27] identified three types of arousal: 1) cognitive arousal related to thinking and attention allocation, 2) affective arousal related to feeling and emotions (both positive and negative), and 3) physical arousal related to physical exertion. Following Cannon, cognitive games should have elicited cognitive arousal, while interactions with Pleo affective arousal.

Concerning the last research question, we expected that EDA features would have been correlated with the items of OME and OERS more characteristic of the two activities attention and cognitive difficulty in the cognitive games, and pleasure and attitude towards the game during robot play.

\section{RESULTS}

We performed a pairwise t-test using SPSS (version 22.0) on the items of OME and OERS obtained during the two activities to check for significant differences. As a first step before analysis, we computed the median of OME and OERS scores obtained across the three sessions of cognitive games, and play with the robot. Results (see Table 2) revealed that cognitive games were perceived as significantly more difficult at a cognitive level $(M=2.07, S D=.730)$ with respect to interactions with Pleo $(M=1.00, S D=.000$, $t(13)=5.491, \quad p<.001)$. Moreover, they outlined that participants felt considerably more pleasure during social robot interactions $(M=3.93, S D=1.141)$ compared to cognitive games $(M=1.93, S D=1.141, t(13)=-5.508, p<.001)$, but were also less alert during interactions with Pleo $(M=4.64, S D=.497)$ with respect to cognitive games $(M=5.00$, $S D=.00, t(13)=2.687, p<.05)$.

As second analysis, we ran a pairwise t-test on the mean differential features of EDA. First, we extracted the differential features of EDA from each session (diffSMA, diffM, diffSD, diffRNG, diffSH, diffNPR, diffKURT, and diffSKEW). Subsequently, we computed the mean differential features of EDA in the three sessions of cognitive games and in the three sessions of robot play (MeanDiffSMA, MeanDiffM, MeanDiffSD, MeanDiffRNG, MeanDiffSH, MeanDiffNPR, MeanDiffKURT, and MeanDiffSKEW). We performed the pairwise t-test using the mean differential features of each condition (cognitive games and robot play).

Results (see Table 2) disclose significant differences between activities in MeanDiffKURT and MeanDiffSKEW. Indeed, MeanDiffKURT was lower during cognitive games $(M=247.299, S D=117.856)$ with respect to robot play $(M=360.041, \quad S D=128.376, \quad t(13)=-2.718, \quad p=.018), \quad$ and similarly MeanDiffSKEW was lower during cognitive games $(M=5.875, S D=2.949)$ compared to robot play $(M=9.326$, $S D=2.678, t(13)=-3.764, p=.002)$.

Subsequently, we performed a Pearson product moment correlation (one-tailed) between the items of the OME and OERS and the features of EDA. In the context of cognitive games, results disclose a significant negative correlation between the item attitude towards the partner and the MeanDiffKURT of EDA $(r(12)=-.497 ; p=.035)$, a significant negative correlation between the item attitude towards the partner and the MeanDiffSKEW of EDA $(r(12)=-.502$; $p=.034)$, and a significant positive correlation between the item pleasure and the MeanDiffRNG of EDA $(r(12)=.539$; $p=.023$ ). On the other side, in the sessions of robot play, results highlighted a significant negative correlation between the item attention and the MeanDiffSKEW of EDA $(r(12)=-$ $.517 ; p=.029$ ), a significant negative correlation between the item attitude towards the game and MeanDiffKURT of EDA $(r(12)=-.513 ; p=.030)$, a significant negative correlation between the item attitude towards the game and the

TABLE 2. Results of the Pairwise t-test on OME and OERS items and EDA features

\begin{tabular}{|c|c|c|c|c|c|c|c|c|}
\hline & \multicolumn{2}{|c|}{ Cognitive games } & \multicolumn{2}{|c|}{ Pleo } & \multicolumn{4}{|c|}{ Paired Differences } \\
\hline OME & $M$ & $S D$ & $M$ & $S D$ & $M$ & $S D$ & t-value & $p$-value \\
\hline Attention & 3.64 & .497 & 3.36 & .929 & .29 & .914 & 1.170 & $>.05$ \\
\hline Attitude game & 5.71 & 1.069 & 5.79 & 1.251 & -.07 & 1.141 & -.234 & $>.05$ \\
\hline Attitude partner & 5.43 & 1.222 & 5.29 & 1.267 & .14 & .949 & .563 & $>.05$ \\
\hline Cognitive difficulty & 2.07 & .730 & 1.00 & .000 & 1.07 & .730 & 5.491 & $* * *<.001$ \\
\hline OERS & $M$ & $S D$ & $M$ & $S D$ & $M$ & $S D$ & t-value & p-value \\
\hline Pleasure & 1.93 & 1.141 & 3.93 & 1.141 & -2.00 & 1.359 & -5.508 & $* * *<.001$ \\
\hline Anger & 1.00 & .000 & 1.29 & .726 & -.29 & .726 & -1.472 & $>.05$ \\
\hline Anxiety/Fear & 1.00 & .000 & 1.00 & .000 & / & l & I & I \\
\hline Sadness & 1.00 & .000 & 1.14 & .535 & -.14 & .535 & -1.000 & $>.05$ \\
\hline Alertness & 5.00 & .000 & 4.64 & .497 & .36 & .497 & 2.687 & $*<.05$ \\
\hline EDA features & $M$ & $S D$ & $M$ & $S D$ & $M$ & $S D$ & t-value & p-value \\
\hline DiffSMA & .0006 & .0003 & .0006 & .0004 & .0001 & .0003 & .585 & $>.05$ \\
\hline DiffM & .2304 & .1202 & .2159 & .1775 & .0145 & .0886 & .613 & $>.05$ \\
\hline DiffSD & .1032 & .0611 & .1103 & .0671 & -.0071 & .0371 & -.720 & $>.05$ \\
\hline DiffRNG & .4532 & .2102 & .4561 & .2162 & -.0029 & .1196 & -.092 & $>.05$ \\
\hline DiffSH & .6038 & .3610 & .6189 & .3560 & -.0151 & .2443 & -.231 & $>.05$ \\
\hline DiffNPR & 2.9295 & .8956 & 2.5086 & 1.2462 & .4209 & .9387 & 1.678 & $>.05$ \\
\hline DiffKURT & 247.2991 & 117.8564 & 360.0415 & 128.3763 & -112.7423 & 155.1860 & -2.718 & $*<.05$ \\
\hline DiffSKEW & 5.8753 & 2.9486 & 9.3260 & 2.6778 & -3.4507 & 3.4299 & -3.764 & $* *<.005$ \\
\hline
\end{tabular}

$*, * *$ and $* * *$ refer to the level of significance of the t-values 
MeanDiffSKEW of EDA $(r(12)=-.590 ; p=.013)$, a significant negative correlation between attitude towards the partner and MeanDiffM $(r(12)=-.561 ; p=.018)$, a significant positive correlation between pleasure and MeanDiffSMA $(r(12)=.483 ; p=.040)$, a significant positive correlation between pleasure and MeanDiffNPR $(r(12)=.577 ; p=.015)$, a significant negative correlation between pleasure and MeanDiffKURT $(r(12)=-.577 ; p=.015)$, and a significant negative correlation between pleasure and MeanDiffSKEW $(r(12)=-.604 ; p=.011)$.

\section{DISCUSSION}

Results from OME and OERS show differences in engagement and affect between the two different activities. Cognitive games elicit significantly higher cognitive exertion (i.e. cognitive difficulty), whereas robot play elicits significantly higher positive affect (i.e. pleasure). These findings are in line with our hypotheses. However, in addition to the hypothesized effects, we found a significantly higher alertness of participants during cognitive games with respect to robot play. This might be due to the fact that cognitive games have a very precise flow and rules to attain at each step of the activity and consequently require participants to be always present and attentive.

Differences in EDA between activities regard mainly the frequency distribution of the signal. The analyses show that the interactions with Pleo are characterized by lower harmonics with respect to cognitive games (skewness) and by a more defined peak of the frequency distribution curve compared to cognitive games (kurtosis). This is in line with our expectations and can be explained with the fact that the two proposed activities both elicit arousal, but of a different type, cognitive and affective.

From the results of the Pearson product moment correlation, it is also evident that MeanDiffKURT and MeanDiffSKEW entertain significant negative correlations with the items of OME and OERS in both conditions: attitude towards the partner in cognitive games, and attention, attitude towards the game and pleasure in robot play. Pleasure is also positively correlated with MeanDiffM in the robot condition, and with EDA features delineating the phasic component of the EDA signal (brisk increases in EDA related to short-term events): MeanDiffRNG in cognitive games, and MeanDiffSMA, and MeanDiffNPR in robot play. In this case, results are not in line with our hypotheses. Indeed, they underline that EDA features correlate with items of OME and OERS more related to attitudes and affective states in both types of activities and not just in robot play.

To summarize, by triangulating the analyses of the data from the different measurement instruments, we found that the engagement with the robot was more pleasurable for the participants and less difficult at a cognitive level. However, it also implied less alertness. Related to EDA patterns, we unveiled that the most positive engagement in both activities was likely to be characterized by an EDA with a higher frequency (lower skewness), a less defined peak of the frequency distribution (lower kurtosis), and a higher phasic activity (high range, SMA and number of peaks ratio).

The present study was mainly limited by the sample size and by its geographical homogeneity (i.e. all participants are from Spain). Ideally, a higher number of participants and a more heterogeneous sample would have made the results more generalizable. However, the collection of quality data in the dementia population is extremely time-consuming due to the amount of work and structuring that it entails. In order to strike a balance between the need for bigger sample sizes and the time expenditure that the collection of field data in people with dementia imply, research teams should work in network and collect data using common procedures.

To conclude, the results of this study open up promising possibilities for research in the field of dementia care. Indeed, they reveal that psychophysiological measures can be used to enrich the measurement of engagement in dementia given that behavior observation is not enough. This is especially beneficial in order to study the psychological and affective states of people with dementia associated apathy who show blunted emotional expressivity on a behavioral level, but also for people with very severe dementia whose behaviors are hard to understand and whose language got lost.

\section{ACKNOWLEDGMENT}

A special thanks for the support goes to the nursing homes Redòs de Sant Josep i Sant Pere (Sant Pere de Ribes, Barcelona) and La Mallola (Esplugues de Llobregat, Barcelona). We would like to thank the directive boards of the nursing homes, the psychologists Anna Barea and Neus Sanchez, the social educators Laia Aranda and Elisabeth Seguer, and, in particular, all the participants of our study.

\section{REFERENCES}

[1] Prince, M., Guerchet, M., \& Prina, M. (2013). The global impact of dementia 2013-2050. Alzheimer's Disease International.

[2] Finkel, S. I., \& Burns, A. (2000). Introduction. International psychogeriatrics, 12(S1), 9-12.

[3] Burgio, L. D., Scilley, K., Hardin, J. M., Janosky, J., Bonino, P., Slater, S. C., \& Engberg, R. (1994). Studying disruptive vocalization and contextual factors in the nursing home using computer-assisted real-time observation. Journal of Gerontology, 49(5), P230-P239.

[4] Brooker, D. J., Woolley, R. J., \& Lee, D. (2007). Enriching opportunities for people living with dementia in nursing homes: an evaluation of a multi-level activity-based model of care. Aging and Mental Health, 11(4), 361-370.

[5] Fossey, J., Ballard, C., Juszczak, E., James, I., Alder, N., Jacoby, R., \& Howard, R. (2006). Effect of enhanced psychosocial care on antipsychotic use in nursing home residents with severe dementia: cluster randomised trial. Bmj, 332(7544), 756-761.

[6] Kolanowski, A., Buettner, L., Litaker, M., \& Yu, F. (2006). Factors that relate to activity engagement in nursing home residents. American journal of Alzheimer's disease and other dementias, 21(1), 15-22.

[7] van der Ploeg, E. S., Eppingstall, B., Camp, C. J., Runci, S. J., Taffe, J., \& O'Connor, D. W. (2013). A randomized crossover trial to study the effect of personalized, one-to-one interaction using Montessoribased activities on agitation, affect, and engagement in nursing home residents with Dementia. International psychogeriatrics, 25(04), 565575.

[8] Gitlin, L. N., Winter, L., Earland, T. V., Herge, E. A., Chernett, N. L., Piersol, C. V., \& Burke, J. P. (2009). The Tailored Activity Program to reduce behavioral symptoms in individuals with dementia: feasibility, acceptability, and replication potential. The Gerontologist, 49(3), 428-439.

[9] Moyle, W., Cooke, M., Beattie, E., Jones, C., Klein, B., Cook, G., \& Gray, C. (2013). Exploring the effect of companion robots on emotional expression in older adults with dementia: A pilot 
randomized controlled trial. Journal of gerontological nursing, 39(5), 46-53.

[10] Šabanović, S., Bennett, C. C., Chang, W. L., \& Huber, L. PARO robot affects diverse interaction modalities in group sensory therapy for older adults with dementia. In 2013 IEEE 13th International Conference on Rehabilitation Robotics (ICORR), 1-6

[11] Robinson, H., MacDonald, B., Kerse, N., \& Broadbent, E. (2013). The psychosocial effects of a companion robot: A randomized controlled trial. Journal of the American Medical Directors Association, 14(9), 661-667.

[12] Cohen-Mansfield, J., Dakheel-Ali, M., \& Marx, M. S. (2009). Engagement in persons with dementia: the concept and its measurement. The American journal of geriatric psychiatry, 17(4), 299-307.

[13] Lawton, M. P., Van Haitsma, K., \& Klapper, J. (1999). Observed emotion rating scale. Journal of Mental Health and Aging, 5(1), 69-81.

[14] Andreassi, J. L. (2013). Psychophysiology: Human behavior \& physiological response. Psychology Press.

[15] Wada, K., Shibata, T., Musha, T., \& Kimura, S. (2005, August). Effects of robot therapy for demented patients evaluated by EEG. In Intelligent Robots and Systems, 2005.(IROS 2005). 2005 IEEE/RSJ International Conference on (pp. 1552-1557). IEEE.

[16] Wada, K., \& Shibata, T. (2008). Social and physiological influences of robot therapy in a care house. Interaction Studies, 9(2), 258-276.

[17] Kawaguchi, Y., Wada, K., Okamoto, M., Tsujii, T., Shibata, T., \& Sakatani, K. (2011, July). Investigation of brain activity during interaction with seal robot by fNIRS. In RO-MAN, 2011 IEEE (pp. 308-313). IEEE.

[18] Treusch, Y., Page, J., van der Luijt, C., Beciri, M., Benitez, R., Stammler, M., \& Marcar, V. L. (2015). Emotional reaction in nursing home residents with dementia-associated apathy: A pilot study. Geriatric Mental Health Care, 3(1), 1-6.

[19] Alam, M. A. U., Roy, N., Holmes, S., Gangopadhyay, A., \& Galik, E. (2016, June). Automated functional and behavioral health assessment of older adults with dementia. In Connected Health: Applications, Systems and Engineering Technologies (CHASE), 2016 IEEE First International Conference on (pp. 140-149). IEEE.

[20] Tiberio, L., Cesta, A., Cortellessa, G., Padua, L., \& Pellegrino, A. R. (2012, September). Assessing affective response of older users to a telepresence robot using a combination of psychophysiological measures. In RO-MAN, 2012 IEEE (pp. 833-838). IEEE.

[21] Henriques, R., Paiva, A., \& Antunes, C. (2013, September). Accessing emotion patterns from affective interactions using electrodermal activity. In Affective Computing and Intelligent Interaction (ACII), 2013 Humaine Association Conference on (pp. 43-48). IEEE.

[22] Leite, I., Henriques, R., Martinho, C., \& Paiva, A. (2013, March). Sensors in the wild: Exploring electrodermal activity in child-robot interaction. In Proceedings of the 8th ACM/IEEE international conference on Human-robot interaction (pp. 41-48). IEEE Press.

[23] Rosenthal-von der Pütten, A. M., Krämer, N. C., Hoffmann, L., Sobieraj, S., \& Eimler, S. C. (2013). An experimental study on emotional reactions towards a robot. International Journal of Social Robotics, 5(1), 17-34.

[24] Reisberg, B., Ferris, S. H., De Leon, M. J., \& Crook, T. (1988). Global deterioration scale (GDS). Psychopharmacol Bull, 24(4), 6613.

[25] Mordoch, E., Osterreicher, A., Guse, L., Roger, K., \& Thompson, G. (2013). Use of social commitment robots in the care of elderly people with dementia: A literature review. Maturitas, 74(1), 14-20.

[26] Garbarino, M., Lai, M., Bender, D., Picard, R. W., \& Tognetti, S. (2014). Empatica E3 - A wearable wireless multi-sensor device for real-time computerized biofeedback and data acquisition. In 2014 EAI 4th International Conference on Wireless Mobile Communication and Healthcare (Mobihealth) 39-42.

[27] Cannon, W. B. (1915). Bodily changes in pain, hunger, fear, and rage: An account of recent researches into the function of emotional excitement. D. Appleton and company. 